\title{
Balkanologie
}

Balkanologie Revue d'études pluridisciplinaires

Vol. VIII, $n^{\circ} 1 \mid 2004$

Volume VIII Numéro 1

\section{Gossiaux [Jean-François], Pouvoirs ethniques dans les Balkans}

Paris : Presses universitaires de France (collection « Ethnologies »),2002, $21 ? \mathrm{p}$.

Gilles de Rapper

\section{CpenEdition}

Journals

Édition électronique

URL : http://journals.openedition.org/balkanologie/2076

DOI : $10.4000 /$ balkanologie.2076

ISSN : 1965-0582

Éditeur

Association française d'études sur les Balkans (Afebalk)

Édition imprimée

Date de publication : 1 juin 2004

ISSN : 1279-7952

Référence électronique

Gilles de Rapper, «Gossiaux [Jean-François], Pouvoirs ethniques dans les Balkans », Balkanologie [En ligne], Vol. VIII, $n^{\circ} 1$ | 2004, mis en ligne le 21 janvier 2010, consulté le 17 décembre 2020. URL : http:// journals.openedition.org/balkanologie/2076 ; DOI : https://doi.org/10.4000/balkanologie.2076

Ce document a été généré automatiquement le 17 décembre 2020.

(c) Tous droits réservés 


\title{
Gossiaux [Jean-François], Pouvoirs ethniques dans les Balkans
}

Paris : Presses universitaires de France (collection « Ethnologies »),2002, $21 ? \mathrm{p}$.

\author{
Gilles de Rapper
}

\section{RÉFÉRENCE}

Gossiaux [Jean-François], Pouvoirs ethniques dans les Balkans, Paris : Presses universitaires de France (collection « Ethnologies »),2002, 21 ? p.

1 L'ouvrage de Jean-François Gossiaux propose une lecture rigoureuse de l'histoire récente des Balkans à la lumière de quelques concepts clés de la réflexion anthropologique, en particulier de celui d'ethnicité. Le livre s'ouvre avec une interrogation sur le statut du fait ethnique dans l'Europe de la fin du XX $X^{e}$ siècle et du début du XXI et en particulier sur ses relations avec le politique dans l'ex-Yougoslavie. Soulignant l'ambivalence de l'adjectif «ethnique» (qui s'applique aussi bien aux guerres qu'à la musique), l'auteur regrette que ce terme tombé dans l'usage courant, et plus encore dans l'usage politique, apparaisse comme tellement évident qu'il ne donne pas matière à débat. C'est donc à une tentative de définition de l'ethnicité et de ses rapports avec le pouvoir qu'invite ce livre.

Les premiers chapitres ( 1 à 3 ) sont consacrés à une mise en place conceptuelle. L'auteur y rappelle le traitement anthropologique des concepts d'ethnie et de nation, en soulignant en particulier l'apport de F. Barth pour le premier et de E. Gellner pour le second ${ }^{1}$. S'il salue la "révolution copernicienne" par laquelle F. Barth, plutôt que de considérer les groupes ethniques en eux-mêmes, aborde la question de leurs relations et de leur interdépendance (avec la question connexe de leurs frontières), il voit dans son individualisme méthodologique une limite de taille, dans la mesure où il tend à présenter le fait ethnique comme une affaire de stratégie ou de choix personnels, occultant ainsi «la dimension contraignante, et même proprement aliénante, de 
l'ethnicité » (p. 14). Le modèle de E. Gellner, longuement expliqué, lui semble mieux tenir la route, mais soulève une interrogation (à laquelle le reste du livre tente de répondre): les nationalismes apparus en Europe orientale dans les années 1990 relèventils du schéma gellnérien au même titre que ceux du XIX ${ }^{e}$ siècle et du début du $\mathrm{XX}^{\mathrm{e}}$ ? L'auteur - tout en confirmant la «valeur universelle » de ce schéma - introduit la notion de « second nationalisme » (p. 61) pour décrire les phénomènes les plus récents. Contrairement à son prédécesseur, celui-ci se manifeste dans des sociétés déjà industrialisées et surtout apparaît comme "régressif » : il cherche à séparer plutôt qu'à rassembler, à empêcher la communication généralisée (en créant de nouvelles langues) plutôt qu'à la favoriser.

3 L'auteur propose par ailleurs un certain nombre de notions que l'on retrouvera dans le reste de l'ouvrage : relation d'ethnicité, rapport social critique, structure ethnique. La relation d'ethnicité est une relation d'identité/altérité, et c'est à ce titre une relation à trois termes : la référence, au moins implicite, à un tiers, est en effet nécessaire pour dire si $\mathrm{x}$ est différent de $y$. C'est ensuite, et l'auteur fait appel ici à une présentation sous forme de formules mathématiques qui a l'avantage de retenir l'attention du lecteur - à moins qu'elle ne le déroute, une relation d'équivalence, qui a pour conséquence qu'un individu ne peut appartenir qu'à un seul groupe ethnique (ce qui n'est pas le cas de l'appartenance à une culture, qui peut être multiple). Relevant d'une logique simple et rudimentaire (p. 18), la relation d'ethnicité peut être au fondement d'une "structure ethnique» (la reconnaissance par la société de plusieurs groupes ethniques) s'il s'y ajoute un interdit matrimonial, par lequel la société est divisée en «classes endogames qui correspondent aux groupes ethniques» (p. 18). Cet interdit matrimonial répond à un principe de non-cumul des "rapports sociaux critiques ", ce qui veut dire que l'échange matrimonial ne peut avoir lieu entre des groupes dont les rapports dessinent déjà des lignes de division au sein de la société. La structure ethnique exige par ailleurs la reconnaissance d'une règle de descendance qui fixe l'appartenance et inscrit le groupe dans la durée, ce que fait aussi, d'une autre manière, le récit des origines que cultive chaque groupe.

4 Soucieux de ne pas proposer un modèle uniquement balkanique du fait ethnique, mais de donner à sa démonstration une portée plus générale, Jean-François Gossiaux s'appuie sur des exemples variés, provenant en particulier d'Afrique et de la France de l'Ancien Régime, qui offrent de stimulantes perspectives comparatives.

5 Les chapitres suivants sont consacrés à la Yougoslavie, de sa formation à son éclatement et à la création de nouveaux États. L'auteur commence par présenter les "contingences historiques» du nationalisme yougoslave jusqu'à la fin de la " première » Yougoslavie, en 1941. Il réfute l'idée d'une Yougoslavie vouée dès le départ à l'éclatement parce que rassemblant deux modèles de sociétés radicalement différents, l'un issu de l'empire austro-hongrois et occidental, l'autre héritier de l'empire ottoman, et donc oriental. Il montre au contraire qu'il a existé un réel nationalisme yougoslave dont le schéma gellnérien rend compte sans difficulté: création d'une langue commune, « invention » d'institutions communes (la zadruga). Il note cependant que, en plus de l'existence concurrente d'un nationalisme croate, le lien qui s'établit entre la monarchie (serbe) et l'Église orthodoxe à la tête du Royaume des Serbes, Croates et Slovènes, constitue un ferment de division politique (chapitre 4). Le chapitre consacré à la seconde Yougoslavie, fédérale et socialiste, et à sa disparition, s'articule autour de l'opposition entre le «principe des nationalités » et le «modèle austro-marxiste », qui 
s'opposent comme cherchant l'un la territorialisation des nationalités, l'autre leur déterritorialisation. L'éclatement de la Yougoslavie marque en fait la victoire du premier, sous la forme de l'ethnonationalisme et au détriment de la démocratie (chapitre 5). La chapitre consacré à la Macédoine montre comment, depuis le début du $\mathrm{XX}^{\mathrm{e}}$ siècle et la République de Kruševo, deux conceptions antinomiques de la nation macédonienne se sont opposées: l'une territoriale, qui rassemble les différentes populations vivant dans la région, et l'autre nationalitaire, qui identifie la nation à la seule ethnie macédonienne (slave). Ces dernières années, avec l'intensification de l'antagonisme entre Macédoniens et Albanais, ont vu la victoire du «principe des nationalités », le pays étant " partagé de facto entre deux entités ethniques » (chapitre 6).

6 Dans les trois chapitres consacrés aux Valaques/Aroumains, qui forment, avec la Macédoine, le deuxième cas de figure envisagé, l'auteur fait d'abord appel au schéma gellnérien pour expliquer l'absence (ou l'échec) de mouvement national aroumain au XIXe siècle: pasteurs, caravaniers ou commerçants, en déplacement et parlant plusieurs langues, les Valaques n'avaient pas besoin de se doter d'une « haute culture » pour participer à "l'univers de communication généralisée de la société industrielle ", puisqu'ils en étaient déjà des acteurs (chapitre 7). Il montre aussi que c'est le pastoralisme - avec ses valeurs et les stéréotypes qui s'y attachent - qui constitue le principal marqueur de la frontière ethnique entre les Valaques et les autres, au moins en Macédoine. Et même lorsqu'il disparaît comme mode de vie, ne laissant plus que les stéréotypes, il reste de la place pour le projet de faire de l'identité valaque un objet politique (chapitre 8). Ce projet est récent (années 1980-1990) et se manifeste dans une nouvelle affirmation de l'identité valaque, qui remplace la «sous-déclaration systématique » de la période précédente, ainsi que par la revendication d'une langue spécifique et commune à tous les Valaques. Cette affirmation possède deux dimensions contradictoires, entre un légitimisme macédonien (la Macédoine est le pays des Valaques, les Valaques sont les meilleurs des Macédoniens) et un «ethnicisme transnational » qui s'efforce de dépasser les frontières de la Macédoine, voire même de trouver dans les institutions européennes et internationales une autorité politique (un « toit politique » dans le langage gellnérien). Cet ethnicisme transnational, qui partage certains traits avec le nationalisme, exclut cependant et la lutte armée et la création d'un État, et peut être mis en relation avec le capitalisme du XXI ${ }^{e}$ siècle, comme le nationalisme l'était avec celui du XIX ${ }^{e}$ siècle, toujours selon Gellner (chapitre 9).

7 Le livre de Jean-François Gossiaux peut ainsi être vu comme une tentative de « mise à jour " du schéma gellnérien à la lumière des événements des années 1990 dans les Balkans. Il est aussi une lecture d'un grand pan de l'histoire européenne à travers le prisme de l'ethnicité, ramenée ici dans sa (dangereuse) simplicité : «L'ethnicité est une façon élémentaire de classer, de représenter, de mettre un ordre simple dans la complexité du monde. Elle constitue l'ordre par excellence, celui qui procède du pouvoir de nommer ». Il est sans doute à peine nécessaire de préciser que l'ensemble de la démonstration est écrit dans une langue claire et précise, et que la richesse et la diversité des sources (observations et entretiens, presse, biographies, ...), tout comme le recours discret mais toujours efficace à l'humour, maintiennent constamment l'attention du lecteur. 


\section{NOTES}

1. Barth (Frederik), ed., Ethnie Groups and Boundaries, Boston: Little Brown, 1969; Gellner (Ernest), Nations and Nationalism, Ithaca : Cornell University Press, 1983. Il faut souligner la grande clarté de l'exposé, qui fait de cette première partie une très bonne introduction aux théories anthropologiques de l'ethnie et de la nation, y compris la théorie (russe) de l'« ethnos ", relativement moins bien connue, mais dont l'influence sur les conceptions en vigueur dans les Balkans ne doit pas être négligée. 